\title{
Acadêmicos da Unochapecó na Interação com Sistema Único de Saúde e ESF através do Projeto Pró-Saúde
}

\author{
Interaction between Students from the \\ Chapecó Community University and the \\ Unified National Health System and Family \\ Health Strategy through the Pró-Saúde \\ Program
}

Pauline Cureau Miechuanski Maria Elisabeth Kleba

PALAVRAS CHAVE:

- Educação Superior;

- Atenção Primária à Saúde;

- Saúde da Família;

- Equipe Multidisciplinar;

- Sistema Único de Saúde.
- Education, Higher;

- Primary Health Care;

- Family Health;

- Multidisciplinary Team;

- Single Health System.
Recebido em: 01/10/2010

Aprovado em: 24/11/2010

\section{RESUMO}

O presente artigo relata reflexões sobre possibilidades e desafios de implantação da Estratégia Saúde da Família em um município do sul do Brasil, a partir da experiência de uma acadêmica da 2 a fase do curso de Medicina da Universidade Comunitária da Região de Chapecó - Unochapecó. A experiência se deu no contexto de um dos projetos aprovados no Pró-Saúde, Vivências Interdisciplinares e Multiprofissionais, que inclui tutorias e atividades de observação no âmbito da atenção básica do município, envolvendo estudantes e professores de dez cursos de graduação e profissionais da rede de serviços da Secretaria de Saúde de Chapecó. A partir dessa observação foram identificadas as seguintes dificuldades: equipe de saúde restrita em relação à diversidade de profissionais, que é necessária para as ações de promoção da saúde na comunidade; baixo comprometimento dos usuários; déficits na relação médico-paciente; e baixo nível de satisfação de alguns profissionais no trabalho. Como avanços foram percebidos o comprometimento da equipe e a aproximação da unidade de saúde com o setor da assistência social em prol do trabalho intersetorial.

This article reports on possibilities and challenges in the implementation of the Family Health Strategy in a municipality in Southern Brazil, based on the experience of a female undergraduate medical student at the Chapecó Community University (Unochapecó). The experience occurred in the context of one of the projects approved by the Educational Program for Health Work (Pró-Saúde), entitled Interdisciplinary and Multi-professional Experiences, which includes tutorships and observation activities in primary care in the municipality, involving students and faculty from ten undergraduate courses and staff from the Chapecó municipal health services. This observation detected the following difficulties: a limited health team in terms of the diversity of professionals, which is necessary for the health promotion activities in the community; low commitment on the part of users; flaws in the physician-patient relationship; and low level of work satisfaction among some of the health professionals. Improvements included greater staff commitment and closer collaboration between the health unit and the social assistance department in favor of inter-sector work. 


\section{INTRODUÇÃO}

O Programa Nacional de Reorientação da Formação Profissional em Saúde (Pró-Saúde), instituído pelos Ministérios da Educação e da Saúde a partir de 2005, tem em vista a interação de acadêmicos com atividades do Sistema Único de Saúde (SUS) a fim de contribuir para a substituição do clássico modelo hospitalocêntrico e biomédico de atendimento à saúde, centrado em enfermidades, para o novo arquétipo de Atenção Básica, o qual visa, principalmente, à promoção e à proteção da saúde.

Especialmente nos cursos de Medicina, "sair do hospital para trabalhar em outros cenários representa um grande desafio" $^{\prime 1}$ (p. 208). Os futuros profissionais resistem a se inserir em cenários que os distanciem do lugar de prática que idealizam, ou seja, hospitais privados ou consultório próprio. O Pró-Saúde preconiza que os cenários de aprendizagem devem possibilitar o desenvolvimento de relações eficazes, favorecendo a aproximação do estudante com a comunidade e a vivência de práticas de acolhimento e da clínica ampliada em saúde.

Tendo aprovado sua proposta nos editais do Pró-Saúde, a Universidade Comunitária da Região de Chapecó - Unochapecó, em parceria com a Secretaria de Saúde de Chapecó (SSA), desenvolve atualmente cerca de 50 projetos, dentre quais se encontra o Vivências Interdisciplinares e Multiprofissionais VIM. O projeto VIM apresenta como objetivo a inserção dos acadêmicos em Unidades de Atenção Básica à Saúde para a observação das atividades realizadas no local, interagindo com profissionais e usuários do sistema, permitindo que aconteça um intercâmbio de ideias e visões que transformarão, ou criarão, a concepção sobre o Sistema Único de Saúde e tudo que o envolve. A atividade de observação é direcionada à compreensão acerca do conceito ampliado da saúde e sua aplicação nos espaços da rede de Atenção Básica à Saúde, identificando possibilidades e desafios para a concretização dos princípios da integralidade e da intersetorialidade. Nesse sentido, a experiência busca potencializar o papel do estudante enquanto agente transformador da realidade social, sensibilizando-o para a compreensão de sua condição de futuro profissional de saúde, membro de uma equipe multiprofissional, com atuação em um determinado território e em um sistema de saúde.

O VIM envolve estudantes dos cursos de Educação Física, Enfermagem, Farmácia, Fisioterapia, Medicina, Nutrição, Odontologia, Psicologia, Serviço Social e Ciências Biológicas da Unochapecó, tendo como tutores profissionais da SSA do município e professores dos cursos citados, além de outros profissionais interessados. Em 2010, a atividade envolveu 270 estudantes, divididos em 29 grupos, 29 tutores e 29 acolhedores (profissionais dos serviços que acolheram e acompanharam as atividades nos Centros de Saúde do município).
Exposições teóricas não se comparam ao entendimento que se pode obter vivenciando a complexa realidade do SUS. Realidade esta que pode ser muitas vezes satisfatória, no entanto, em outras ocasiões, pode encontrar-se, lamentavelmente, distante do aceitável.

Este artigo traz reflexões de uma acadêmica da 2a fase do curso de Medicina da Unochapecó a partir de sua experiência em um grupo do VIM. As considerações são realizadas com base nos depoimentos de usuários do SUS, agentes comunitários de saúde, auxiliares e técnicos de enfermagem e administrativos, e enfermeiros. São elaboradas, ainda, análises durante o acompanhamento de consultas médicas e odontológicas, bem como da equipe da Estratégia Saúde da Família (ESF), durante seu percurso para os atendimentos domiciliares aos usuários na comunidade.

\section{DESENVOLVIMENTO}

As atividades do VIM incluem cinco momentos tutoriais, nos quais os estudantes são incentivados à leitura e ao debate, problematizando suas ideias e experiências sobre o SUS e reconhecendo em documentos e publicações aspectos relacionados à teoria e à prática da gestão, do ensino e do cuidado em saúde. Após a terceira tutoria, os estudantes realizam 12 horas de observação em um dos Centros de Saúde do município de Chapecó, sendo acolhidos por um dos membros da equipe local de saúde. O conhecimento dos Centros de Saúde não envolve somente seu espaço físico e as atividades que ocorrem em seu interior, mas compreende toda sua área de abrangência e território, envolvendo as características econômicas, culturais e sociais da população local, bem como os serviços e as formas de organização existentes. Esses dados revelam-se de extrema importância para o planejamento de ações de promoção, proteção e recuperação da saúde e para a lida com os pacientes, suas famílias e a comunidade de forma geral.

As atividades práticas realizadas na unidade permitem constatações, dentre elas a de que, apesar de haver vários profissionais da saúde empenhando-se para que o atendimento na unidade seja o melhor possível, seria de grande auxílio a presença de outros profissionais, tais como nutricionistas, psicólogos e educadores físicos, que, embora sejam considerados da área da saúde, ainda lutam por seu espaço no SUS junto ao atendimento básico. Essas orientações visam prioritariamente à promoção e à prevenção da saúde e auxiliariam na mudança do modelo biomédico, o qual, ao longo dos anos, reduziu a análise médica a um nível celular e molecular e, por conseguinte, o ser humano passou a ser decomposto em compartimentos. As enfermidades não são vistas como resultantes da interação de um todo indivisível, podendo ser influenciadas pelo estado 
psíquico e emocional do paciente e pelo meio onde ele vive, seus costumes, cultura e as pessoas com as quais se relaciona. Ações terapêuticas que não levam em consideração tais pressupostos acabam, muitas vezes, por curar uma patologia sem interferir nas causas e/ou na dinâmica que a desencadeou, mantendo o indivíduo vulnerável ao desenvolvimento ou à manifestação de outros sinais e sintomas patológicos ${ }^{2}-$ es- $^{-}$ pectro da maioria dos médicos de especialidades, atualmente. Mostra-se extremamente necessária a visão que engloba todo o indivíduo; e a presença desses ofícios, certamente, aliviaria a grande carga de atendimentos médicos e também a demanda por medicamentos como antidepressivos, além de promover uma melhor qualidade de vida para a população.

É possível citar algumas iniciativas da atual política de saúde que buscam impulsionar mudanças no modelo assistencial e, como consequência, na prática dos profissionais da saúde. A primeira foi a promulgação do atendimento integral à saúde como uma das diretrizes constitucionais3, reiterada como princípio da integralidade na Lei 8.0804. Esse princípio orienta ainda a organização da Atenção Básica, que tem a Saúde da Família como estratégia prioritária, e assume como fundamento, dentre outros, "efetivar a integralidade em seus vários aspectos, a saber: integração de ações programáticas e de demanda espontânea; articulação das ações de promoção à saúde, prevenção de agravos, vigilância à saúde, tratamento e reabilitação, trabalho de forma interdisciplinar e em equipe, e coordenação do cuidado na rede de serviços" ${ }^{\prime 5}$ (p. 11).

Em 2008, o Ministério da Saúde aprova a Portaria 154, instituindo os Núcleos de Apoio à Saúde da Família (Nasf), com o objetivo de apoiar, ampliar e aperfeiçoar a atenção e a gestão na Atenção Básica e na Saúde da Família. Constituído por equipes que incluem profissionais de diferentes áreas do conhecimento, os Nasf devem atuar no apoio e em parceria com as equipes da ESF, incluindo "ações intersetoriais e interdisciplinares, promoção, prevenção, reabilitação da saúde e cura, além de humanização de serviços, educação permanente, promoção da integralidade e da organização territorial dos serviços de saúde" ${ }^{\prime \prime}$ (p. 10-11).

Relatos de administradores das unidades, médicos e enfermeiros permitiram notar, ainda, que a real procura por um atendimento de clínica geral revela-se, significativamente, escassa. Grande parte da população busca não uma atenção primária à saúde, objetivo principal da Atenção Básica, e sim procura o serviço de saúde com o intuito de solicitar encaminhamentos a especialistas, exames laboratoriais ou, ainda, prescrição de medicamentos, sem ao menos esperar pela consulta do médico previamente.
Pode ser que essa contrariedade relativa ao atendimento médico nas Unidades de Saúde tenha sido gerada pela antiga visão hospitalocêntrica, a qual mostrava-se centralizadora e discriminatória ${ }^{7}$, cujas atividades estavam centradas nos hospitais. A alta demanda de atendimento nesses locais e a carência de profissionais fizeram com que os atendimentos se tornassem inapropriados pela insuficiência de atenção fornecida aos indivíduos e pela distância existente na relação médico-paciente - devida, talvez, ao excesso de solicitação de exames complementares de diagnóstico, por exemplo, os laboratoriais. Os exames complementares tomaram o lugar dos físicos, os quais se mostram o elo entre o médico e o paciente, enfraquecendo a confiança dos cidadãos em relação aos médicos.

Atualmente, diversos profissionais na Unidade Básica de Saúde lidam com a falta de reconhecimento do seu trabalho, o que acarreta a falta de desejo de trabalhar no Sistema Único de Saúde, o que, dentre outros motivos, ocasiona a alta taxa de rotatividade de profissionais no sistema tão criticada pelos usuários. A insuficiência de profissionais pode originar a desordenada troca de funções, quando alguns tomam as atividades de outros, o que gera um transtorno ainda maior. Outro fator que esmorece a pretensão dos médicos a serem funcionários do SUS é a ausência de plano de carreira para essa profissão. Iniciar em um cargo com uma determinada remuneração sem expectativas de avanços faz com que os médicos busquem se desligar do sistema, seja por conta de alguma atividade complementar, seja pela aderência a programas de saúde privativos ou atividade autônoma.

Em estudo realizado no município de Botucatu, constatou-se dentre outros fatores de desestímulo à permanência dos profissionais médicos na ESF a baixa realização profissional, além do pouco reconhecimento por parte da população e de outros médicos. Isso provoca a alta rotatividade, já anteriormente mencionada, e dificulta a criação de vínculo com a população, resultando na não adesão desta às práticas de acompanhamento regular, perpetuando, assim, a cultura dos atendimentos eventuais. ${ }^{8}$

Os depoimentos obtidos nas atividades de observação revelaram que a ausência de um número significativo de usuários em atendimentos e exames marcados agrava as filas do SUS e denota certo descaso com os direitos da coletividade, na medida em que aumentam a demanda reprimida por assistência, impedindo o uso por outros indivíduos que necessitariam de atendimento mais urgente. Isso ainda faz com que os faltantes tenham de remarcar o atendimento, estendendo cada vez mais a fila de espera e, consequentemente, sobrecarregando o sistema. 
Essa situação permite a reflexão de que, talvez, os entraves existentes no SUS não sejam infundidos apenas pela carência de profissionais, espaço, materiais ou medicamentos, mas também pelo descomprometimento de muitos indivíduos que não estão cientes de que além de possuírem seus direitos no sistema, possuem deveres e responsabilidades. A Lei 8.080 (Art $2^{\circ}, \S 2$ ) articula claramente que "o dever do Estado não exclui o das pessoas, da família, das empresas e da sociedade" ${ }^{\prime 4}$. Por exemplo, a maioria dos usuários não tem conhecimento de que pode e necessita participar diretamente das decisões adotadas nas unidades por meio dos Conselhos Locais de Saúde, espaço de diálogo com a comunidade existente em todos os Centros de Saúde do município de Chapecó desde 1997. Infelizmente, a maior parte dos indivíduos nem ao menos conhece a existência do conselho. Essa falha deixa de lado o preceito de que o SUS deve ser uma organização que envolve um processo decisório participativo e que a Atenção Básica deve desenvolver relações de vínculo e responsabilização entre as equipes e a população adscrita, garantindo continuidade das ações e a longitudinalidade do cuidado em saúde ${ }^{5}$.

O vínculo se constrói como compromisso partilhado entre equipe de trabalhadores e usuários na produção de cuidado. Essa interação possibilita apoio à autonomia do usuário para viver sua vida, aumentando sua capacidade de autocuidado, de utilização do próprio poder e de estabelecimento de compromissos e contratos com os outros.

A dedicação das unidades em relação aos seus grupos de risco, como hipertensos, diabéticos, gestantes e idosos, revela um dos avanços significativos do SUS. Calendários especiais de atendimentos, organização de grupos de apoio com palestras periódicas, em que todos os profissionais integrantes da ESF têm seu espaço para prestar orientações e apoio à população, manifestam-se como ações de vultosa estima para a promoção e proteção da saúde.

OSUS, além dos atendimentos realizados na unidade pela ESF, conta com o apoio de outras instituições de peso vinculadas ao Ministério do Desenvolvimento Social e de Combate à Fome, entre outras, as quais auxiliam o Ministério da Saúde nos suprimentos das carências da população e na melhoria da qualidade de vida. Uma dessas instituições é o Centro de Referência de Assistência Social (Cras), unidade pública da política de assistência social, de base municipal, integrante do Sistema Único de Assistência Social (Suas), localizado em áreas com maiores índices de vulnerabilidade e risco social. O Cras se destina à prestação de serviços e programas socioassistenciais, na perspectiva de potencializar a proteção social básica às famílias e indivíduos ${ }^{9}$. No território observado, percebeu- -se uma importante articulação desse serviço com o Centro de Saúde local com vistas à atuação intersetorial.

A vivência realizada pelo grupo de estudantes no Centro de Saúde possibilitou compartilhar informações, percepções e sentimentos, primeiramente nos pequenos grupos tutoriais e, posteriormente, em evento de socialização, no qual estavam presentes os demais atores envolvidos na experiência (estudantes, professores, profissionais e gestores da rede de serviços de saúde de Chapecó), bem como outros interessados na proposta. Nesse grande debate, foi possível refletir que todos fazem parte desse processo em construção, em que o SUS constitui-se vivo e pulsante, está em constante mudança e aprimoramento, tanto de suas estruturas e dos seus serviços, quanto de seus atores - trabalhadores, gestores e usuários.

\section{CONSIDERAÇÕES FINAIS}

A partir da interação entre acadêmicos, Sistema Único de Saúde e toda sua abrangência, permite-se inferir o quão importante se mostra o conhecimento de seus serviços, pois, partindo da dinâmica da comunidade e do próprio Sistema, podem-se analisar as carências, falhas e aspectos positivos do SUS, COnhecimentos que auxiliarão os futuros profissionais formados. A integração entre acadêmicos de diversos cursos da área da saúde com toda a complexidade das condições de saúde no Brasil, desde o princípio da trajetória universitária, possibilita uma troca de concepções e contribui para que se possam analisar aspectos do SUS a serem aperfeiçoados e, a partir de ações dos estudantes e futuros profissionais, seja desenvolvida a capacidade de cooperação para essa evolução.

O Sistema Único de Saúde revela-se incipiente diante de toda a história da saúde em nosso país. Assim, o sistema se encontra distante de sua total concretização. Possui seus limites e possibilidades, mostrando-se necessário adaptar-se, acompanhando as mudanças sociais, econômicas e culturais da população brasileira. A alta demanda por atendimentos nas Unidades Básicas de Saúde e a precariedade das condições de vida de muitos brasileiros impedem que sejam realizadas apenas promoção e proteção à saúde dos cidadãos, afastando os princípios da Atenção Primária à Saúde da realidade.

A experiência do VIM suscitou muitas reflexões, base para aprofundar e ampliar os conhecimentos teórico-práticos ao longo do curso. Pode-se notar que a situação atual se revela extremamente transformada, considerando-se apenas duas décadas atrás. É possível ter esperanças de que, futuramente, o SUS terá um funcionamento muito mais adequado à situação brasileira. Entretanto, para que isso ocorra, não se depende apenas de suas leis e atos burocráticos, mas sua organização encontra-se sujeita a ações de colaboração dos pro- 
fissionais que nele estão inseridos e, ainda, da cooperação dos próprios usuários. Cogitar, nesse momento, um sistema que se mostre realmente integral e universal em todos os níveis de assistência e complexidade, considerando-se todo o capital e a participação necessária para sua ocorrência, revela-se apenas uma utopia.

\section{REFERÊNCIAS}

1. Ferreira RC, Fiorini VML, Crivelaro E. Formação profissional no SUS: o papel da Atenção Básica em Saúde na perspectiva docente. Rev Bras Educ Med. 2010;34(2): 207-15.

2. Capra F. O Ponto de Mutação. São Paulo: Cultrix, 2001.

3. Brasil. Constituição da República Federativa do Brasil: promulgada em 05 de outubro de 1988.

4. Brasil. Lei no 8.080, de 19 de setembro de 1990. Dispõe sobre as condições para a promoção, proteção e recuperação da saúde, a organização e o funcionamento dos serviços correspondentes e dá outras providências. Diário Oficial [da República Federativa do Brasil], Brasília 19 de setembro de 1990.

5. Brasil. Ministério da Saúde. Portaria № 648, de 28 de março de 2006. Aprova a Política Nacional de Atenção Básica, estabelecendo a revisão de diretrizes e normas para a organização da Atenção Básica para o Programa Saúde da Família (PSF) e o Programa Agentes Comunitários de Saúde (PACS). Disponível em <http: / / dtr2004.saude.gov. $\mathrm{br} /$ dab/docs/legislacao/portaria_648_28_03_2006.pdf> Acesso em 23 abr. 2009.

6. Brasil. Ministério da Saúde. Diretrizes dos NASF. Brasília: Ministério da Saúde/Secretaria de Atenção Básica/Depar- tamento de Atenção Básica, 2009. (Cadernos de Atenção Básica, n.27)

7. Minayo MCS. Os 20 anos do SUS e os avanços na vigilância e na proteção à saúde. Epidemiol Serv Saúde. 2008;17(4): 245-446.

8. Goncalves RJ, Soares RA, Troll T, Cyrino EG. Ser médico no PSF: formação acadêmica, perspectivas e trabalho cotidiano. Rev. Bras. Educ. Med. 2009;33(3): 382-392.

9. Brasil. Ministério do Desenvolvimento Social e de Combate a Fome. Norma Operacional Básica - NOB/SUAS: construindo as bases para a implantação do Sistema Único de Assistência Social. Brasília: Ministério do Desenvolvimento Social e de Combate a Fome/Secretaria Nacional de Assistência Social, 2005.

\section{CONTRIBUIÇÃO DOS AUTORES}

Todos os autores participaram forma suficiente na redação do texto, concepção e desenho deste estudo, da análise e interpretação dos dados assim como da redação deste texto.

\section{CONFLITO DE INTERESSES}

Declarou não haver.

\section{ENDEREÇO PARA CORRESPONDÊNCIA}

Pauline Cureau Miechuanski

Rua Tenente Camargo, 1191

Presidente Kennedy - Francisco Beltrão

CEP 85605-090 PR

E-mail: pauline.m@unochapeco.edu.br 